\title{
Efeito do treinamento físico em diabéticos tipo I: revisão sistemática de ensaios clínicos randomizados
}

\author{
Effect of physical training on type I diabetics: systematic review of randomized \\ controlled trials
}

\section{AUTHOR'S \\ Roselaine da Silva Gomes ${ }^{1,2}$ (1) \\ Cassiano Ricardo Rech ${ }^{2}$ (D) \\ Clóvis Arlindo de Sousa ${ }^{1}$ (D) \\ 1 Universidade Regional de Blumenau. \\ Departamento de Educação Física, Blumenau, SC, \\ Brasil. \\ 2 Universidade Federal de Santa Catarina. Centro \\ de Desportos, Florianópolis, SC, Brasil.}

\section{CORRESPONDING}

Roselaine da Silva Gomes

roselaine.s.gomes@gmail.com

Rua das Missões 275, Bairro Ponta Aguda,

Blumenau, SC, Brasil, CEP: 89051000.

DOI

10.12820/rbafs.v.22n5p422-28

\begin{abstract}
RESUMO
O exercício físico (EF) tem sido recomendado no controle da diabetes tipo 1 (DM1), porém, seus efeitos na hemoglobina glicosilada (HbA1c), dose de insulina e perfil lipídico, ainda são controversos. $\mathrm{O}$ objetivo deste estudo foi analisar as evidências do EF em adolescentes com DM1, por meio de uma revisão sistemática. Realizou-se uma busca nas bases de periódicos PubMed, Biblioteca Virtual de Saúde e EBSCOHost. Foram incluídos estudos clínicos controlados e randomizados que testaram o efeito do EF na HbA1c, na dose de insulina e em marcadores do perfil lipídico em adolescentes com DM1, publicados em português ou inglês, entre 2000 e 2017. Foram incluídos cinco estudos em jovens de 10 a 16 anos. As intervenções foram com exercícios aeróbicos, exercícios combinados e pilates, em períodos de três a seis meses. A frequência foi uma a três vezes/semana, com sessões de 45 a 120 minutos e intensidades entre 40-85\% da frequência cardíaca máxima (FCMáx), 80-90\% da frequência cardíaca de reserva e 50-100\% de 10 repetições máximas (RM). Apenas um estudo mostrou-se eficaz na melhora da HbA1c, dois na dose de insulina e quatro no perfil lipídico. Um estudo foi efetivo na $\mathrm{HbA1c}$, na dose de insulina e no perfil lipídico simultaneamente, incluindo exercícios aeróbicos, de flexibilidade, neuromusculares e de equilíbrio, com intensidade de $65-85 \%$ FCMáx e 50-100\% de 10 RM. Os resultados apontam que não existe consistência nos achados. Conclui-se que não há evidência científica suficiente e consistente do tipo, frequência, intensidade e duração dos programas de EF para adolescentes com DM1.
\end{abstract}

Palavras-chave: Exercício físico; Diabetes mellitus tipo1; Revisão.

\begin{abstract}
Exercise has been recommended for the control of type 1 diabetes mellitus (T1DM), but its effects on glycosylated hemoglobin $(H b A 1 c)$, insulin dose and lipid profile are still controversial. This study aims to analyze evidence of exercise in adolescents with T1DM, through a systematic review. The search was carried out in the bases of periodicals PubMed, Biblioteca Virtual de Saúde and EBSCOHost. Controlled clinical trials and randomized studies were included that showed results on the effect of exercise on $\mathrm{HbA1c}$, insulin dose and lipid profile in T1DM adolescents, published in Portuguese or English between the period 2000-2017. Were included five studies with adolescents in the age of 10 to 16. The interventions were with aerobic, combined exercises and pilates during the period of three to six months. The frequency was of one to three times/week with sessions of 45-120 min. Intensities ranged from 40-85\% of the Maximum Heart Rate, 80-90\% of the Heart Rate of Reservation and 50-100\% of 10 Maximum Reps. One study was effective in HbA1c, two in the insulin dose and four in the lipid profile. Only one study was effective in improvement of theHbA1c, insulin dose and lipid profile simultaneously, including aerobic exercises, flexibility, neuromuscular and balance with intensity of 65-85\% Maximum Heart Rate and 50-100\% of 10 Maximum Reps. The results indicate that there is no consistency in the findings. We conclude that there is insufficient and consistent scientific evidence of the type, frequency, intensity, and duration of exercise programs for adolescents with T1DM.
\end{abstract}

Keywords: Exercise; Diabetes mellitus type 1; Review.

\section{Introdução}

A diabetes mellitus tipo 1 (DM1) é uma patologia autoimune que se caracteriza, principalmente, pela perda progressiva das funções das células Beta-pancreáticas promovendo um desequilíbrio metabólico grave ${ }^{1,2}$. As Diretrizes da Sociedade Brasileira de Diabetes (20152016) $)^{3}$ indicaram acentuada variação geográfica da in- cidência de DM1 em jovens com menos de 15 anos de idade. Em São Paulo, entre 1990 e 1994, observou-se uma incidência de oito casos por 100 mil habitantes/ ano ${ }^{4}$. Já em Córdoba (Argentina), foi de sete casos por 100 mil habitantes/ $\mathrm{ano}^{5}$, no mesmo período.

Os efeitos da DM1 na saúde são relatados na literatura $^{6-8}$. Indivíduos com DM1 apresentam compli- 
cações micro e macrovasculares como, por exemplo, retinopatia, nefropatia e neuropática diabética ${ }^{6-8}$,que afetam diretamente o modo de vida dessa população. Estudos clínicos indicaram que a prática regular de exercício físico (EF) possui efeito no controle glicêmico em pessoas com diabetes mellitus tipo 2 (DM2), indicando redução da hemoglobina glicosilada (HbA1c) a longo prazo $^{9-12}$. Entretanto, ainda são controversos os resultados do efeito do EF na saúde de jovens com $\mathrm{DM} 1^{13}$. Um estudo conduzido com adolescentes mostrou efeito positivo do $\mathrm{EF}$ na $\mathrm{HbA} 1 \mathrm{c}^{13}$; mas em outro os efeitos foram inconclusivos ${ }^{14}$.

Assim, esse estudo tem como objetivo analisar as evidências científicas sobre o efeito do EF na HbA1c, dose de insulina e perfil lipídico de adolescentes com DM1, por meio de uma revisão sistemática de estudos clínicos randomizados. Espera-se que essa revisão inclua estudos recentes e que tenham utilizado parâmetros mínimos para seleção dos participantes e controle da intervenção, além de analisar os possíveis vieses de qualidade dos artigos incluídos na revisão, para que se possa sintetizar os achados sobre a relação entre EF e DM1 em adolescentes.

\section{Métodos}

O presente estudo seguiu os procedimentos metodológicos estabelecidos pelo guia PRISMA (Principais Itens para Relatar Revisóes Sistemáticas e Meta-análises) $)^{15}$. Após uma ampla busca para identificação dos descritores mais adequados ao objetivo deste estudo, optou-se por utilizar os Descritores em Ciências da Saúde (DeCS). Os termos foram testados individualmente e combinados. Utilizou-se a seguinte combinação "exercise" OR "training" AND "randomized controlled trial" AND "type 1 diabetes mellitus".

A busca foi realizada nas bases PubMed, Biblioteca Virtual de Saúde (BVS) e EBSCOHost. Foram incluídos estudos de natureza quantitativa (estudos clínicos controlados e randomizados), que apresentassem resultados sobre o efeito do EF na HbA1c, na dose de insulina e no perfil lipídico, em adolescentes com DM1, publicados em português ou inglês. A busca delimitou-se entre o período de 2000-2017 e foi realizada em maio de 2017.

A busca abrangeu três etapas: leitura dos títulos, resumos e textos completos, realizadas de modo independente, por dois avaliadores (RSG e CAS). Em caso de divergência uma terceira opinião foi tomada (CRR). Foram adotados como critérios de inclusão: a) artigos publicados nos últimos 17 anos; b) amostra de adolescentes com diagnóstico de DM1; c) intervenção somente com EF; d) estudos com delineamento clínico controlado e randomizado; e) apenas estudos que investigaram o efeito crônico do EF foram incluídos; f) análise de pelo menos um efeito do exercício na $\mathrm{HbA1c}$, dose de insulina ou perfil lipídico da amostra.

Os títulos selecionados nas bases de periódicos $(\mathrm{n}=$ 1.190), foram importados para o software Zotero, onde foram excluídos os títulos duplicados $(\mathrm{n}=193)$.Optou-se por exportar para o Excel os dados $(n=997)$, para leitura dos títulos, resumos e posteriormente o texto completo. Destes, 579 foram excluídos por que a amostra era composta por indivíduos com DM2, $(\mathrm{n}=259)$ pela intervenção ou pelo fato do objetivo principal do estudo não ser o $\mathrm{EF},(\mathrm{n}=88)$ não envolveram pessoas com DM1 e a intervenção não ser EF, (n=61), a intervenção ser apenas uma sessão de EF ou inferior a oito semanas, e $(n=5)$ por ser de caráter experimental, não apresentar um grupo controle,estudo com animais, população adulta ou crianças.

Após a seleção dos artigos foram extraídas as seguintes informações: autor, ano de publicação, local do estudo, faixa etária, tamanho e sexo dos participantes, período de estudo, frequência e duração do $\mathrm{EF}$, efeitos da intervenção na $\mathrm{HbA1c}$, na dose de insulina e no perfil lipídico pré e pós intervenção.

A qualidade dos estudos incluídos na revisão foi analisada segundo ferramenta da colaboração Cochrane ${ }^{16}$ considerando cinco domínios: a) geração de sequência aleatória; b) ocultação de alocação; c) desfechos incompletos; d) relato de desfecho seletivo e;e) outras fontes de viés. Para cada domínio o pesquisador identificou o estudo como "baixo risco", "risco incerto" e "alto risco", em relação ao viés. Dois pesquisadores (RSG e CAS) realizaram a avaliação de modo independente e em caso de dúvidas outra opinião foi tomada (CRR).

Para análise dos resultados das intervenções foi considerado o efeito do EF em três desfechos: a) HbA1c; b) na dose de insulina e; c) no perfil lipídico. Esses desfechos foram selecionados por serem importantes marcadores de saúde em pessoas com DM1. Para considerar um efeito significativo da intervenção as estatísticas testadas deveriam apresentar níveis de significância com valores de $\mathrm{p}<0,05$. O tamanho do efeito da intervenção nos desfechos também foi calculado.

\section{Resultados}

A busca inicial resultou em 1.190 títulos. Após a exclusão dos artigos duplicados, leitura dos títulos e resumos, foram selecionados 42 estudos para leitura na íntegra. Um total de cinco estudos atenderam aos critérios de inclusão (Figura 1). 
A maior parte dos estudos incluídos na revisão foi desenvolvido em jovens com idade entre 10 e 16 anos, com amostras com 16 e 196 indivíduos. Três estudos foram realizados em países da Europa (Turquia ${ }^{17}$, Bélgi$\mathrm{ca}^{18}$ e França ${ }^{14}$ ) e dois na África (Arábia Saudita ${ }^{19}$ e Egito $\left.^{13}\right)$. Quanto ao tipo de EF, foram identificadas intervenções com exercícios aeróbicos ${ }^{19}$ ou combinados ${ }^{13,14,18}$ e intervenção com pilates ${ }^{14}$. O período das intervenções variou de três a seis meses, com frequência de uma e três vezes por semana, com sessões de 45 a 120 minutos de duração. $\mathrm{O}$ controle da intensidade foi realizado pelo método da frequência cardíaca $(\mathrm{FC})$ e teste de uma repetição máxima (RM). Para a $\mathrm{FC}$, foram observadas intensidades entre 40 e $85 \%$ da frequência cardíaca máxima (FCMáx), 80 a 90\% da frequência cardíaca de reserva (FCR). Para as atividades de força foi observada uma intensidade entre 50 a 100\% de 10 RM (Tabela 1).

$\mathrm{O}$ efeito das intervenções variou de acordo com os desfechos de saúde analisados. No total, cinco estudos ${ }^{13,14,17-19}$ testaram os efeitos das intervenções nos níveis de hemoglobina glicosilada (HbA1c), mas apenas um apresentou efeito positivo ao final da intervenção $(p=0,01)^{13}$. A intervenção realizada em um período de seis meses, com três sessões semanais e com duração de aproximadamente, 65 minutos, e exercícios aeróbios, resistidos, de flexibilidade, neuromusculares e equilíbrio, mostrou-se efetiva na redução dos valores de $\mathrm{HbA1} \mathrm{c}^{13}$. As demais foram avaliadas ou não mostraram efeitos

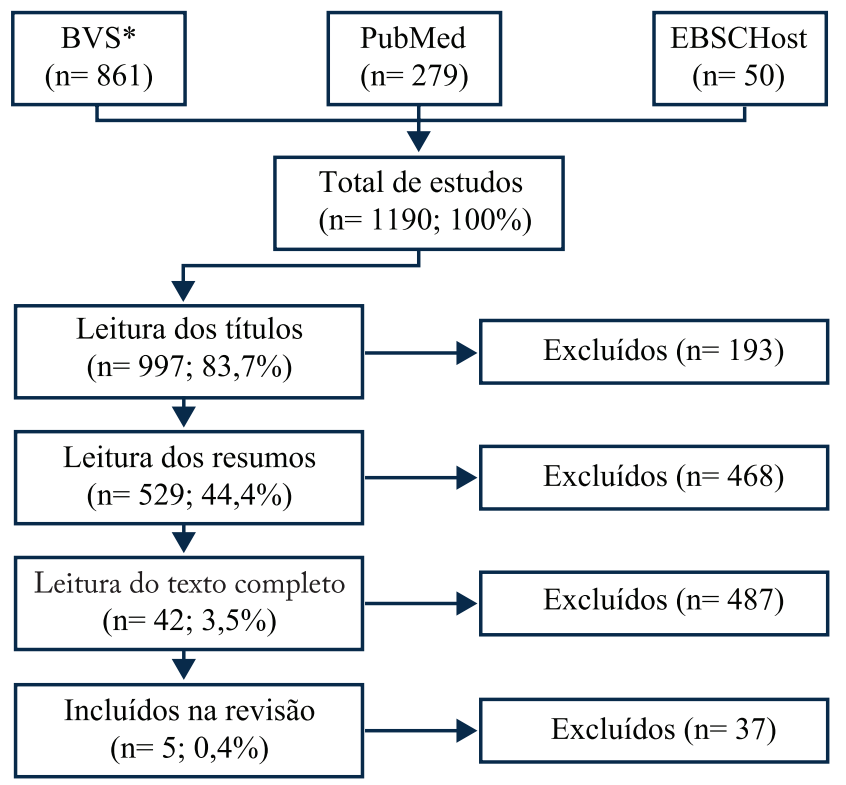

Figura 1 - Fluxograma de identificação dos estudos clínicos controlados e randomizados que avaliaram o efeito do exercício em adolescentes com diabetes mellitus tipo 1 , incluídos na revisão sistemática seguindo as orientações do PRISMA. significativos na HbA1c ( $>0,05)$ (Tabela 1$)$.

Em cinco estudos, o efeito da intervenção foi testado na dose de insulina dos indivíduos com DM1 $1^{13,14,17-19}$. Destes, apenas dois reportaram reduções significativas $^{13,18}$. Um programa de EF com duração de cinco meses, com frequência de duas vezes por semana e sessões de 50 minutos de exercícios aeróbios, com intensidade de 65 a 85\% da FC, e exercícios resistidos, foi eficaz na redução da insulina $(p<0,05)^{18}$. Outro estudo mostrou resultado semelhante com uma intervenção realizada por um período de seis meses. O programa de EF era composto por exercícios aeróbios com intensidade variando entre 65 e $85 \%$ da frequência cardíaca máxima, os resistidos tinham intensidade entre 50 a $100 \%$ de 10 $\mathrm{RM}$, além de exercícios de flexibilidade, neuromusculares e de equilíbrio ${ }^{21}$. Este programa de exercício mostrou-se efetivo para diminuição da dose de insulina quando realizado uma $(\mathrm{p}=0,02)$ e três vezes por semana $(\mathrm{p}<0,01)^{13}$.

Em relação ao perfil lipídico, quatro intervenções foram analisadas, porém apenas uma apresentou alterações significativas ${ }^{13}$. O programa que contempla mais aptidões físicas, composto por exercícios aeróbios, resistidos, de flexibilidade, neuromusculares e de equilíbrio apresentou efeito positivo no perfil lipídico, quando realizado uma ou três vezes por semana,respectivamente, aumentando os valores da lipoproteína de alta densidade (HDL) ( $p=0,01$ e $p<0,01)$ e diminuindo os valores da lipoproteína de baixa densidade (LDL) $(\mathrm{p}=0001$ e $\mathrm{p}=0,001)$, dos triglicerídeos $(\mathrm{TG})(\mathrm{p}<0,01$, ambos) e do colesterol total (CT) $(\mathrm{p}=0,01)^{13}$.

Quanto à avaliação da qualidade dos estudos, foi observado que para nenhum dos itens avaliados foi identificado um alto risco de viés (Tabela 2). Porém, em todos os estudos foram identificados itens com informações pouco detalhadas que podem prejudicar julgamento preciso da qualidade das informações e resultados dos estudos.

\section{Discussão}

Os resultados da revisão apontam que são escassas as evidências sobre a relação entre DM1 e EF em estudos clínicos controlados e randomizados em adolescentes. Esses resultados não permitem identificar uma consistência dos achados, especialmente devido à diversidade das intervenções, que possuem distintos métodos de controle e determinação de volume e intensidade do EF. De modo geral, existem evidências de melhora nos níveis de $\mathrm{HbA1c}$, na dose de insulina e no perfil lipídico de indivíduos com DM1 que realizam EF, porém esses achados ainda são inconsistentes. 
Tabela 1 - Estudos inclusos na revisão sistemática e respectivas variáveis analisadas após intervenção de exercício em adolescentes com DM1.

\begin{tabular}{|c|c|c|c|c|c|c|}
\hline Estudos & Heyman et al. ${ }^{14}$ & Salem et $\mathrm{al}^{13}$ & Salem et a ${ }^{13}$ & Dhooge et a ${ }^{18}$ & Tunar et $\mathrm{a}^{17}$ & Tomar et $\mathrm{al}^{19}$ \\
\hline Sexo (n) & $\mathrm{F}(\mathrm{n}=7)$ & F e $M(n=75)$ & F e $M(n=73)$ & F e $M(n=8)$ & $\mathrm{F}$ e $M(\mathrm{n}=17)$ & $M(n=12)$ \\
\hline Intervenção & $\begin{array}{l}\text { a) exercício aeróbio } \\
\text { ( } 80-90 \% \text { da FCR). } \\
\text { b) exercício de } \\
\text { força. } \\
\text { c) Atividade física } \\
\text { não supervisionada. }\end{array}$ & $\begin{array}{l}\text { a) exercício aeróbio } \\
\text { ( } 65-85 \% \text { FCMáx.). } \\
\text { b) exercício resistido } \\
\text { ( } 50-100 \% \text { de } 10 \mathrm{RMs}) \text {. } \\
\text { c) exercícios de flexibilidade, } \\
\text { neuromusculares e de } \\
\text { equilíbrio. }\end{array}$ & $\begin{array}{l}\text { a) exercício aeróbio ( } 65-85 \% \\
\text { FCMáx.). } \\
\text { b) exercício resistido } \\
(50-100 \% \text { de } 10 \mathrm{RMs}) \text {. } \\
\text { c) exercícios de flexibilidade, } \\
\text { neuromusculares e de } \\
\text { equilíbrio. }\end{array}$ & $\begin{array}{l}\text { a) exercício aeróbio } \\
\text { (60-75\% da } \\
\text { FCMáx.). } \\
\text { b) exercício resistido } \\
\text { (\% } 1 \mathrm{RM}) \text {. }\end{array}$ & $\begin{array}{l}\text { a) exercício de } \\
\text { pilates }\end{array}$ & $\begin{array}{l}\text { a) exercício } \\
\text { aeróbio: } 1-4 \\
\text { semana }(40-0 \% ; \\
\text { semana 5-8 } \\
(50-60 \%) ; \text { semana } \\
\text { 9-12 (60-70\%) da } \\
\text { FCMáx. }\end{array}$ \\
\hline Período & 6 meses & 6 meses & 6 meses & 5 meses & 3 meses & 3 meses \\
\hline $\begin{array}{l}\text { Frequência e } \\
\text { duração }\end{array}$ & $\begin{array}{l}120 \mathrm{~min} . \text { Sup. } \\
2 \mathrm{x} / \mathrm{sem} . \\
+60 \mathrm{~min} . \\
1 \mathrm{x} / \mathrm{sem}\end{array}$ & $\begin{array}{c} \pm 65 \mathrm{~min} \\
1 \mathrm{x} / \mathrm{sem}\end{array}$ & $\begin{array}{l} \pm 65 \mathrm{~min} \\
3 \mathrm{x} / \mathrm{sem}\end{array}$ & $\begin{array}{l}50 \mathrm{~min} \\
2 \mathrm{x} / \mathrm{sem}\end{array}$ & $\begin{array}{l}45 \mathrm{~min} \\
3 \mathrm{x} / \mathrm{sem}\end{array}$ & $\begin{array}{l}60 \mathrm{~min} . \\
3 \mathrm{x} / \mathrm{sem}\end{array}$ \\
\hline $\begin{array}{l}\text { Idade } \\
\text { (média } \pm d p)\end{array}$ & $15,9 \pm 1,5$ & $14,7 \pm 2,2$ & $14,5 \pm 2,4$ & $\begin{array}{c}14,1 \\
(10,1-16,8)^{*}\end{array}$ & $14,3 \pm 1,8$ & $14,27 \pm 1,73$ \\
\hline $\mathrm{HbA1c}$ & $8,0 \pm 1,2$ & $8,1 \pm 1,1$ * & $7,8 \pm 1,1^{*}$ & $\begin{array}{c}7,8 \\
(6,7-10,0)^{*}\end{array}$ & $8,8 \pm 1,5$ & $\begin{array}{c}11,3 \\
(8,7-12,7)\end{array}$ \\
\hline $\begin{array}{l}\Delta \text { efeito } \\
\text { (p-valor) }\end{array}$ & & $-0,57(0,03)^{*}$ & $-0,79(0,01)^{*}$ & & $-0,06(>0,05)$ & \\
\hline Insulina & $1,00 \pm 0,18$ & $1,1 \pm 0,26^{*}$ & $0,09 \pm 0,3$ & $\begin{array}{c}0,90 \\
(0,79-1,52)^{*}\end{array}$ & $1,0 \pm 0,2$ & 0,80 \\
\hline $\begin{array}{l}\Delta \text { efeito } \\
\text { (p-valor) }\end{array}$ & ---- & $-0,20(0,002)^{*}$ & - 0,75 (0,001) & --- & $-0,33(>0,05)$ & 0,$6 ; 1,2(0,385)$ \\
\hline HDL & $1,52 \pm 0,31$ & $56,1 \pm 12,5$ * & $48,6 \pm 3,9^{* * * *}$ & & $56,9 \pm 9,6$ & $\begin{array}{c}48 \\
(37-76)\end{array}$ \\
\hline $\begin{array}{l}\Delta \text { efeito } \\
\text { (p-valor) }\end{array}$ & $-0,28(>0,05)$ & $0,77(0,01)^{*}$ & $2,29(0,00)^{* * * *}$ & --- & $0,26(>0,05)$ & --- \\
\hline LDL & $2,45 \pm 0,58$ & $89,0 \pm 33,4$ & $102,7 \pm 27$ & --- & $85,3 \pm 14,6$ & $\begin{array}{c}101 \\
(69-129)\end{array}$ \\
\hline $\begin{array}{l}\Delta \text { efeito } \\
\text { (p-valor) }\end{array}$ & $-1,11(>0,05)$ & $-0,32(0,01)$ & $-0,87(0,001)^{* * * *}$ & --- & $-0,12(<0,05)$ & --- \\
\hline TG & $0,63 \pm 0,10$ & $109,4 \pm 36,9$ & $135,6 \pm 30,7^{* *}$ & --- & $89,9 \pm 46,8$ & $\begin{array}{c}66 \\
(43-207)\end{array}$ \\
\hline $\begin{array}{l}\Delta \text { efeito } \\
\text { (p-valor) }\end{array}$ & $-0,10(>0,05)$ & $-0,30\left(0,00^{* *}\right)$ & $-1,09(0,00)^{* *}$ & --- & $0,109>0,05)$ & --- \\
\hline $\mathrm{CT}$ & $5,21 \pm 0,41$ & $153,0 \pm 44,3$ & $145,0 \pm 27$ & --- & $167,5 \pm 25,8$ & $\begin{array}{c}160 \\
(120-217)^{*}\end{array}$ \\
\hline $\begin{array}{l}\Delta \text { efeito } \\
\text { (p-valor) }\end{array}$ & $-0,95(>0,05)$ & $-0,34(0,01)^{*}$ & $-1,88(0,00)^{* *}$ & --- & $0,00(>0,05)$ & --- \\
\hline
\end{tabular}

M: masculino; F: feminino. HbA1c: hemoglobina glicosilada. HDL: lipoproteína de alta densidade. LDL: -lipoproteína de baixa densidade. TG: triglicerídeos. TC: colesterol total. FC: Frequência cardíaca. FCMáx.: Frequência cardíaca máxima. FCR: Frequência cardíaca de repouso. RM: Repetição Máxima. *p $\leq 0,05$, ** $\leq 0,001$.

Revisões sistemáticas realizadas sobre a relação entre EF e DM1 mostraram benefícios na saúde dos indivíduos mais ativos, porém, ainda não está clara a relação existente entre o tipo, volume e intensidade do $\mathrm{EF}^{20,21}$. Nessa revisão, dentre os cinco estudos analisados, apenas uma intervenção mostrou efeito positivo na mudança dos níveis de $\mathrm{HbA} 1 \mathrm{c}$, de dose de insulina e em marcadores do perfil lipídico ${ }^{13}$ de modo simultâneo (Tabela 3).

Essa intervenção caracterizou-se como mista, incluindo exercícios aeróbicos, de flexibilidade, neuromusculares e de equilíbrio realizados em intensidade moderada a vigorosa (65 e 85\% FCMáx e 50-100\% de 10 RM). Acredita-se que devido ao elevado volume (seis meses com um total de 195 minutos/semana), os resultados podem ter sido mais evidentes. Além disso, o tempo total semanal em EF de intensidade moderada e vigorosa foi superior às recomendações da Organização Mundial de Saúde para adultos jovens ( $>150$ minutos/semana) $)^{22}$. Nesse mesmo estudo, os resultados para aqueles indivíduos que realizaram apenas 65 minutos/semana de EF foram menores quando comparados com aqueles que realizaram maior volume de EF. Assim, pode-se inferir que adotar EF de intensidades moderadas e vigorosas, com duração próxima a $200 \mathrm{mi}-$ nutos/semana ou 30 minutos/dia podem promover benefícios significativos a saúde dos jovens com DM1. Isso vai ao encontro das recomendações da Organização Mundial da Saúde para faixa etária dos cinco aos dezessete anos são de 60 minutos/dia de EF de intensidade moderada e vi- 
Tabela 2 - Avaliação do risco de viés dos estudos segundo ferramenta da colaboração Cochrane dos estudos clínicos controlados e randomizados que avaliaram o efeito do exercício em adolescentes com diabetes mellitus tipo 1, incluídos na revisão sistemática.

\begin{tabular}{|c|c|c|c|c|c|}
\hline $\begin{array}{l}\text { Domínio } \rightarrow \\
\text { Estudo } \downarrow\end{array}$ & $\begin{array}{l}\text { Geração de sequência } \\
\text { aleatória }\end{array}$ & Ocultação de alocação & Desfechos incompletos & $\begin{array}{l}\text { Relato de desfecho } \\
\text { seletivo }\end{array}$ & Outras fontes de viés \\
\hline Heyman et $\mathrm{al}^{14}$ & Incerto & Incerto & Baixo & Baixo & Baixo \\
\hline D’hooge et $\mathrm{al}^{18}$ & Baixo & Baixo & Baixo & Incerto & Baixo \\
\hline Salem et al ${ }^{13}$ & Incerto & Incerto & Incerto & Baixo & Incerto \\
\hline Tunar et $\mathrm{al}^{17}$ & Baixo & Incerto & Incerto & Baixo & Baixo \\
\hline Tomar et $\mathrm{al}^{19}$ & Incerto & Incerto & Incerto & Incerto & Baixo \\
\hline
\end{tabular}

Baixo/Incerto - Classificação do risco de viés.

Tabela 3 - Estudos inclusos na revisão sistemática e efeito do exercício nas variáveis que obtiveram diferenças significativas após intervenção em adolescentes com DM1.

\begin{tabular}{|c|c|c|c|c|c|c|c|c|c|c|}
\hline \multirow{2}{*}{ Autor } & \multirow{2}{*}{ Ano } & \multirow{2}{*}{ Local } & \multirow{2}{*}{$\begin{array}{l}\text { No por Grupos/ } \\
\text { Sexo }\end{array}$} & \multirow{2}{*}{ Intervenção } & \multirow{2}{*}{$\mathrm{HbA1c}$} & \multirow{2}{*}{$\begin{array}{l}\text { Dose de } \\
\text { insulina }\end{array}$} & \multicolumn{4}{|c|}{ Perfil lipídico } \\
\hline & & & & & & & HDL & LDL & $\mathrm{TG}$ & $\mathrm{TC}$ \\
\hline \multirow[b]{2}{*}{ Salem et $\mathrm{al}^{13}$} & \multirow[b]{2}{*}{2010} & \multirow[b]{2}{*}{ Egito } & $\mathrm{GI}_{1}-75(\mathrm{~F} / \mathrm{M})$ & $\begin{array}{l}\text { AF supervisionada. EF aeróbico ( } 65 \\
\text { a } 85 \% \text { da FC M). EF resistido ( } 50 \text { a }\end{array}$ & $\sqrt{ }$ & $\sqrt{ }$ & $\sqrt{ }$ & $\sqrt{ }$ & $\sqrt{ }$ & $\sqrt{ }$ \\
\hline & & & $\mathrm{GI}_{2}-73(\mathrm{~F} / \mathrm{M})$ & $\begin{array}{l}100 \% \text { de } 10 \mathrm{RM} \text { ). AF de flexibilidade, } \\
\text { neuromuscular e de equilíbrio. }\end{array}$ & $\sqrt{ } \sqrt{ }$ & $\sqrt{ } \sqrt{ }$ & $\sqrt{ } \sqrt{ }$ & $\sqrt{ } \sqrt{ }$ & $\sqrt{ } \sqrt{ }$ & $\sqrt{ } \sqrt{ }$ \\
\hline D'hooge et al ${ }^{18}$ & 2011 & Bélgica & $\mathrm{GI}-8(\mathrm{~F} / \mathrm{M})$ & $\begin{array}{l}\text { AF supervisionada. EF aeróbico ( } 60 \text { a } 75 \% \\
\text { da FCMáx.). EF resistido (\% } 1 \text { RM - não } \\
\text { descrito o valor específico). }\end{array}$ & - & $\sqrt{ }$ & - & - & - & - \\
\hline Tomar et $\mathrm{al}^{19}$ & 2014 & $\begin{array}{l}\text { Arábia } \\
\text { Saudita }\end{array}$ & $\mathrm{GI}-12(\mathrm{M})$ & $\begin{array}{l}\text { AF supervisionada. AF aeróbica - } \\
\text { Semana 1-4: } 40 \text { a } 50 \% \text { da FCMáx.; } \\
\text { Semana 5-8: } 50 \text { a } 60 \% \text { da FCMáx.; } \\
\text { Semana 9-12: } 60 \text { a } 70 \% \text { da FCMáx. }\end{array}$ & - & - & - & - & - & $\sqrt{ }$ \\
\hline
\end{tabular}

HbA1c - hemoglobina glicosilada, HDL - lipoproteína de alta densidade, LDL - lipoproteína de baixa densidade, TG - triglicerídeos, TC - colesterol total, F - Feminino, M - Masculino, GC - Grupo Controle, GI - Grupo Intervenção, GI - Grupo Intervenção 1, GI - Grupo Intervenção 2, FC - Frequência Cardíaca, FCMáx

- Frequência Cardíaca Máxima, FCR - Frequência Cardíaca de Repouso, RM - Repetição Máxima, $\mathrm{X}$ - vezes, sem - semana, min - minutos, $\sqrt{ }$ p $\leq 0,05, \sqrt{ } \sqrt{ }$ p $\leq 0,001$.

gorosa, totalizando 420 minutos $/$ semana $^{23}$. Os resultados apresentados são suportados por evidências que apontam que exercícios de intensidade moderada e vigorosa são mais efetivos na saúde de indivíduos com DM1 ${ }^{18,19}$.

Observa-se que programas compostos por EFs variados, que recrutem maior repertório motor são mais eficazes na redução da $\mathrm{HbA1c}$ de crianças e adolescentes com DM1 ${ }^{24,25}$. Programas de EF variados e diferenciados podem ser mais eficazes para DM1 em virtude da manifestação da patologia ocorrer em crianças e adolescentes. A elaboração de programas que envolvam esportes e brincadeiras podem ser mais atrativos, estimulando a prática de EF de maneira prazerosa. Ademais, existe ainda uma importante barreira para adoção da prática de EF nessa população, devido ao risco de hipoglicemia ${ }^{26}$. Propor programas de EF relacionados com o cotidiano do jovem pode promover maior adesão e aderência ${ }^{27}$.

$\mathrm{O}$ EF de intensidade vigorosa de modo intermitente promove um menor declínio da glicose sanguínea, pois estimula respostas hormonais e metabólicas antagonistas a diminuição da glicemia. Assim, por mais que eleve os níveis de lactato, pode-se ter um efeito positivo contribuindo na atenuação do declínio da glicemia por inibição da ação da insulina ${ }^{1}$. Além disso, o aumento das catecolaminas em resposta o EF intenso intermi- tente estimula a produção de glicose hepática, ao mesmo tempo em que inibe o consumo de glicose mediado pela insulina ${ }^{1}$. Estudos clínicos controlados ${ }^{28,29}$, que mostraram os efeitos de programas de EFc omposto por jogos esportivos, natação, brincadeiras em crianças e adolescentes obesos, mostraram que um programa realizado duas vezes/semana 100 minutos/semana obteve resultados positivos na concentração de insulina em jejum e o colesterol total ${ }^{28}$. Outro programa realizado três vezes/semana por 210 minutos/semana promoveu reduções significativas na insulina de jejum, triglicerídeos, LDL, HDL e a resistência a insulina ${ }^{29}$.

Quatro estudos não mostraram efeito positivo do EF sobre a $\mathrm{HbA1} \mathrm{c}^{14,17-19}$. Uma das intervenções avaliou o efeito de exercícios de pilates ${ }^{17} \mathrm{e}$ outra intervenção avaliou o efeito de exercícios aeróbios ${ }^{19} \mathrm{e}$ não foram efetivos sobre a HbA1c. O período relativamente curto, de três meses, dessas intervenções pode ter influenciado a ausência desse efeito, assim como a variação da intensidade e o tamanho da amostra. $\mathrm{O}$ fato da intervenção não ter sido supervisionada e as sessões não serem combinadas com outros exercícios podem ser consideradas limitações. A ausência do controle do estilo de vida, sobretudo a ingestão alimentar, também foram consideradas limitações dos estudos. Outra limitação mencionada foi a alteração na nutrição e 
na rotina de insulina, assim como o medo de hipoglicemia, que fez com que os participantes evitassem alguns movimentos e intensidades do programa de exercícios, principalmente nas primeiras semanas ${ }^{17,19}$. Os autores discutem que um período maior de intervenção com exercícios, após a fase de adaptação, pode apresentar resultados diferentes.

Quanto às intervenções com exercícios combinados aeróbios e resistidos, embora realizadas por um período de seis meses, talvez não tenham influenciado a $\mathrm{HbA} 1 \mathrm{c}^{14,18} \mathrm{em}$ virtude do pequeno número da amostra e menor diversidade de atividades físicas para crianças e adolescentes. A intensidade dos exercícios resistidos não foi descrita ${ }^{14}$. Outras limitações envolvem a baixa frequência semanal, a redução da dose de insulina e aumento da ingestão de carboidratos relacionados a episódios de hipoglicemia que ocorreram não só durante os treinos, mais também 12 horas após ${ }^{18}$.

Pode-se sugerir que programas de exercícios combinados, esportes e atividades diversificadas que recrutam um maior repertório motor apresentam efeitos positivos quando praticados por crianças e adolescentes com DM1, sendo que esses benefícios podem ser ainda maiores quando realizados com intensidade moderada e com frequência de três vezes por semana. Considerando a faixa etária de diagnóstico de DM1, pode-se sugerir que os programas de exercícios devem ser atrativos para que haja empenho e interesse dos praticantes à sua realização. A sugestão é a utilização de esportes, atividades com música e brincadeiras para compor o programa de exercício para crianças e adolescentes com DM1.

Uma das principais limitações dessa revisão foi o número reduzido de estudos clínicos randomizados e controlados que tenham utilizado apenas o programa de EF como intervenção, outros estudos randomizados têm incluído outras formas de intervenção como controle da alimentação ou de medicamentos, porém, ao incluir apenas intervenções com EF é possível identificar que é preciso um maior número de estudos sobre o tema. Esse pequeno número de estudos e a falta de consistência dos achados, não permite estabelecer uma metanálise dos resultados. Porém, os resultados da revisão mostram os artigos publicados em importantes bases de periódicos e revisados por pares, além de apresentar uma avaliação da qualidade dos estudos inclusos. Embora o número de artigos inclusos seja baixo, apenas cinco, trata-se do que tem disponível atualmente sobre o tema. Em uma metanálise sobre DM1 e saúde, os autores apontaram que amostras pequenas dificultavam as recomendações ou relatórios mais seguros sobre o efeito do EF na DM1 ${ }^{30}$.
Pode-se concluir que ainda não há evidência científica suficiente e consistente sobre o tipo, frequência, intensidade e duração dos programas de EF para pessoas com DM1. Porém, os resultados sugerem que programas de EF combinados (esportes e atividades diversificadas, jogos, recreativos), que recrutam um maior repertório motor quando executados com intensidade moderada $\mathrm{e}$ vigorosa por um volume maior de 150 minutos/semana e pelo menos três vezes na semana aumentam a aptidão cardiorrespiratória em crianças e adolescentes com DM1 e podem ser importante estratégia para promoção de qualidade de vida dessa população.

\section{Conflito de interesses}

Os autores declaram não haver conflito de interesses.

\section{Financiamento}

A pesquisa foi financiada com recursos próprios dos autores.

\section{Contribuição dos autores}

Gomes RS, participou da concepção inicial do estudo, redação e responsável pela busca da literatura. Rech $\mathrm{CR}$, foi responsável pela revisão crítica, análise e interpretação dos dados. Sousa CA, participou da concepção inicial do estudo, análise, interpretação dos dados e responsável pela busca da literatura.

\section{Agradecimentos}

Agradecemos aos docentes e acadêmicos do programa de extensão "Educação em Saúde: doce alegria da assistência integral" da Universidade de Blumenau (FURB) e aos servidores da Policlínica Universitária e do Hospital Universitário da FURB, onde pudemos realizar as atividades de saúde com as crianças e os adolescentes com diabetes e viabilizar esta revisão sistemática.

\section{Referências}

1. Miculis CP, Mascarenhas LP, Boguszewski MC, Campos W de. Atividade física na criança com diabetes tipo 1. J Pediatr. 2010;86(4):271-8.

2. Fernandes APM, Pace AE, Zanetti ML, Foss MC, Donadi EA. Fatores imunogenéticos associados ao diabetes mellitus do tipo 1. Rev Lat Am Enfermagem. 2005;13(5):743-9.

3. Milech A, Angelucci AP, Golbert A, Matheus A, Carrilho ALF, Ramalho AC, et al. Diretrizes da Sociedade Brasileira de Diabetes (2015-2016). São Paulo: A. C. Farmacêutica; 2016.

4. Ferreira SR, Franco LJ, Vivolo MA, Negrato CA, Simoes AC, Ventureli CR. Population-based incidence of IDDM in the state of São Paulo, Brazil. Diabetes Care. 1993;16(5):701-4.

5. De Beaufort $\mathrm{C}$. Incidence and trends of childhood type 1 diabetes worldwide 1990-1999. Diabet Med J Br Diabet Assoc. 2006;23(8):857-66.

6. Prasanna Kumar KM, Azad K, Zabeen B, Kalra S. Type 1 diabetes in children: Fighting for a place under the sun. Indian J Endocr Metab. 2012;16,Suppl S1:1-3. 
7. Naseri M, Rostami S, Dashtebozorgi B. E ect of Group Training on Glycemic Control in Patients With Diabetes Type-I. Jundishapur J Chronic Dis Care. 2015;4(4):e29728.

8. Rostami S, Parsa-Yekta Z, Naja Ghezeljeh T, Vanaki Z. Supporting adolescents with type 1 diabetes mellitus: A qualitative study. Nurs Health Sci. 2014;16(1):84-90.

9. Pai L-W, Li T-C, Hwu Y-J, Chang S-C, Chen L-L, Chang $\mathrm{P}-\mathrm{Y}$. e e ectiveness of regular leisure-time physical activities on long-term glycemic control in people with type 2 diabetes: a systematic review and meta-analysis. Diabetes Res Clin Pract. 2016;113:77-85.

10. Praet SFE, Van Rooij ESJ, Wijtvliet A, Boonman-de Winter LJM, Enneking T, Kuipers H, et al. Brisk walking compared with an individualised medical tness programme for patients with type 2 diabetes: a randomised controlled trial. Diabetologia. 2008;51(5):736-46.

11. Church TS, Blair SN, Cocreham S, Johannsen N, Johnson $\mathrm{W}$, Kramer K, et al. E ects of aerobic and resistance training on hemoglobin A1c levels in patients with type 2 diabetes: a randomized controlled trial. Jama. 2010;304(20):2253-62.

12. Chen S-C, Ueng K-C, Lee S-H, Sun K-T, Lee M-C. E ect of t'ai chi exercise on biochemical pro les and oxidative stress indicators in obese patients with type 2 diabetes. J Altern Complement Med. 2010;16(11):1153-9.

13. Salem MA, AboElAsrar MA, Elbarbary NS, ElHilaly RA, Refaat YM. Is exercise a therapeutic tool for improvement of cardiovascular risk factors in adolescents with type 1 diabetes mellitus? A randomised controlled trial. Diabetol Metab Syndr. 2010;2(1):2-47.

14. Heyman E, Toutain C, Delamarche P, Berthon P, Briard D, Youssef $\mathrm{H}$, et al. Exercise training and cardiovascular risk factors in type 1 diabetic adolescent girls. Pediatr Exerc Sci. 2007;19(4):408-19.

15. Moher D, Liberati A, Tetzla J, Altman DG. Preferred reporting items for systematic reviews and meta-analyses: the PRISMA statement. PLoS Med. 2009;6(7):e1000097.

16. De Carvalho APV, Silva V, Grande AJ. Avaliação do risco de viés de ensaios clínicos randomizados pela ferramenta da colaboração Cochrane Diagnóstico e Tratamento. 2013;18(1):38-44.

17. Tunar M, Ozen S, Goksen D, Asar G, Bediz CS, Darcan S. Effects of Pilates on metabolic control and physical performance in adolescents with type 1 diabetes mellitus. J Diabetes Complications. 2012;26(4):348-51.

18. D'hooge R, Hellinckx T, Van Laethem C, Stegen S, De Schepper J, Van Aken S, et al. In uence of combined aerobic and resistance training on metabolic control, cardiovascular tness and quality of life in adolescents with type 1 diabetes: a randomized controlled trial. Clin Rehabil.2011;25(4):349-59.

19. Tomar R, Hamdan M, Al-Qahtani MH. E ect of low to moderate intensity walking and cycling on glycaemic and metabolic control in type 1 diabetes mellitus adolescent males: A randomized controlled trial. Isokinet Exerc Sci. 2014;22(3):237-43.
20. Yardley JE, Hay J, Abou-Setta AM, Marks SD, McGavock J. A systematic review and meta-analysis of exercise interventions in adults with type 1 diabetes. Diabetes Res Clin Pract. 2014;106(3):393-400.

21. Tonoli C, Heyman E, Roelands B, Buyse L, Cheung SS, Berthoin S, et al. E ects of di erent types of acute and chronic (training) exercise on glycaemic control in type 1 diabetes mellitus. Sports Med. 2012;42(12):1059-80.

22. WHO. Global recommendations on physical activity for health [Internet]. World Health Organization. [citado 9 de junho de 2017]. Disponível em: http://www.who.int/ dietphysicalactivity/factsheet_recommendations/en/

23. WHO. Physical activity and young people [Internet]. World Health Organization. [citado 9 de junho de 2017]. Disponível em: http://www.who.int/dietphysicalactivity/ factsheet_young_people/en/

24. Ruzic L, Sporis G, Matkovic BR. High volume-low intensity exercise camp and glycemic control in diabetic children. J Pediatr Child Health. 2008;44(3):122-8.

25. Campaigne BN, Gilliam TB, Spencer ML, Lampman RM, Schork MA. E ects of a physical activity program on metabolic control and cardiovascular fitness in children with insulin-dependent diabetes mellitus. Diabetes Care. 1984;7(1):57-62.

26. Leclair E, De Kerdanet M, Riddell M, Heyman E. Type 1 diabetes and physical activity in children and adolescents. J Diabetes Metab S. 2013;10:1-10.

27. Armstrong MJ, Sigal RJ. Physical activity clinical practice guidelines: what's new in 2013? Can J Diabetes. 2013;37(6):363-6.

28. Meyer AA, Kundt G, Lenschow U, Schu -Werner P, Kienast W. Improvement of early vascular changes and cardiovascular risk factors in obese children after a six-month exercise program. J Am Coll Cardiol. 2006;48(9):1865-70.

29. Savoye M, Shaw M, Dziura J, Tamborlane WV, Rose $\mathrm{P}$, Guandalini C, et al. E ects of a weight management program on body composition and metabolic parameters in overweight children: a randomized controlled trial. JAMA. 2007;297(24):2697-704.

30. Nielsen PJ, Hafdahl AR, Conn VS, LeMaster JW, Brown SA. Meta-analysis of the e ect of exercise interventions on tness outcomes among adults with type 1 and type 2 diabetes. Diabetes Res Clin Pract. 2006;74(2):111-20.

Recebido: 16/05/2016 Aprovado: 30/10/2017

\section{Como citar esse artigo:}

Gomes RS, Rech CR, Sousa CA. Efeito do treinamento físico em diabéticos tipo I: revisão sistemática de ensaios clínicos randomizados. Rev Bras Ativ Fís Saúde. 2017; 22(5):422-28. DOI: 10.12820/rbafs. v.22n5p422-28. 